\title{
Running of the coupling and quark mass in SU(2) with two adjoint fermions
}

\author{
Francis Bursa* \\ University of Cambridge, UK \\ E-mail: fwb22@cam.ac.uk

\section{Luigi Del Debbio} \\ University of Edinburgh, $U K$ \\ E-mail: luigi.del.debbioled.ac.uk
}

\section{Liam Keegan}

University of Edinburgh, UK

E-mail: liam.keegan@ed.ac.uk

\section{Claudio Pica}

University of Edinburgh, $U K$

E-mail: claudio.pica@ed.ac.uk

\section{Thomas Pickup}

University of Oxford, $U K$

E-mail: pickup@thphys.ox.ac.uk

We simulate $S U(2)$ gauge theory with two massless Dirac fermions in the adjoint representation. We calculate the running of the Schrödinger Functional coupling and the renormalised quark mass over a wide range of length scales. The running of the coupling is consistent with the existence of an infrared fixed point (IRFP), and we find $0.07<\gamma<0.56$ at the IRFP, depending on the value of the critical coupling.

The XXVII International Symposium on Lattice Field Theory

July 26-31, 2009

Peking University, Beijing, China

\footnotetext{
*Speaker.
} 


\section{Introduction}

The symmetry breaking in technicolor theories is communicated to the Standard Model by a further interaction at some higher energy scale $M$. There is a tension on the value of $M$ : on the one hand $M$ needs to be large so that FCNC interactions are suppressed, on the other it needs to be small to generate the heavier quark masses. The effective operator for the quark masses is:

$$
\mathscr{L}=\frac{1}{M^{2}}\langle\Phi\rangle \bar{\psi} \psi,
$$

where $\psi$ indicates the quark field, and $\Phi$ the field which is responsible for electroweak symmetry breaking. In traditional technicolor models $\Phi=\bar{\Psi} \Psi$ is the chiral condensate of techniquarks. The coefficient in Eq. (1.1) is the chiral condensate at the scale $M$ :

$$
\left.\langle\bar{\Psi} \Psi\rangle\right|_{M}=\left.\langle\bar{\Psi} \Psi\rangle\right|_{\Lambda} \exp \left[\int_{\Lambda}^{M} \frac{d \mu}{\mu} \gamma(\mu)\right],
$$

where $\Lambda$ is the technicolor scale. This suggests a possible way to resolve the tension on $M$ : if $\gamma$ is approximately constant and large over a sufficiently long range in energies, then the condensate will be enhanced. This scenario is known as walking technicolor. Gauge theories with a large number of fermions, or with fermions in higher-dimensional representations of $S U(N)$ [1], are candidates. These theories could have a genuine IR fixed point (IRFP), or simply be close to one.

The existence of an IRFP is a difficult problem to address since it requires quantitative computations in a strongly-interacting theory. Lattice simulations provide first-principle results that can help in determining the phenomenological viability of these models. A number of theories have been studied recently: SU(3) with 8, 10, 12 flavors of fermions in the fundamental representation, $\mathrm{SU}(3)$ with fermions in the sextet representation, and SU(2) with fermions in the adjoint representation. Existing simulations of the Schrödinger functional have identified a possible fixed point in all the above-mentioned theories by noticing a flat behaviour of the running coupling.

In this work we focus on $\mathrm{SU}(2)$ with 2 adjoint flavours, and compute the running coupling in the SF scheme. We also compute the running of the mass, and extract the anomalous dimension.

\section{Basic formulation}

We define the running coupling $\bar{g}^{2}$ non-perturbatively using the Schrödinger Functional method [2, 3]. This is defined on a hypercubic lattice of size $L$, with boundary conditions chosen to impose a background electric field on the system. The spatial link matrices at $t=0$ and $t=L$ are set to:

$$
\left.U(x, k)\right|_{t=0}=\exp \left[\eta \tau_{3} a / i L\right] \quad,\left.\quad U(x, k)\right|_{t=L}=\exp \left[(\pi-\eta) \tau_{3} a / i L\right],
$$

with $\eta=\pi / 4$ [4]. The fermion fields obey

$$
P_{+} \psi=0, \bar{\psi} P_{-}=0 \text { at } t=0 \quad, \quad P_{-} \psi=0, \bar{\psi} P_{+}=0 \text { at } t=L,
$$

where the projectors are defined as $P_{ \pm}=1 / 2\left(1 \pm \gamma_{0}\right)$. The fermion fields also satisfy periodic spatial boundary conditions [5]. We use the Wilson plaquette gauge action, and Wilson fermions in the adjoint representation, as implemented in Ref. [6]. 
The coupling constant is defined as

$$
\bar{g}^{2}=k\left\langle\frac{\partial S}{\partial \eta}\right\rangle^{-1}
$$

with $k=-24 L^{2} / a^{2} \sin \left(a^{2} / L^{2}(\pi-2 \eta)\right)$ chosen such that $\bar{g}^{2}=g_{0}^{2}$ to leading order in perturbation theory. This is a non-perturbative definition of the coupling which depends on only one scale, $L$.

To measure the running of the quark mass, we calculate the pseudoscalar density renormalisation constant $Z_{P}$. Following Ref. [7], $Z_{P}$ is defined by:

$$
Z_{P}(L)=\sqrt{3 f_{1}} / f_{P}(L / 2)
$$

where $f_{1}$ and $f_{P}$ are the correlation functions involving the boundary fermion fields $\zeta$ and $\bar{\zeta}$ :

$$
\begin{aligned}
f_{1} & =-1 / 12 L^{6} \int d^{3} u d^{3} v d^{3} y d^{3} z\left\langle\bar{\zeta}^{\prime}(u) \gamma_{5} \tau^{a} \zeta^{\prime}(v) \bar{\zeta}(y) \gamma_{5} \tau^{a} \zeta(z)\right\rangle, \\
f_{P}\left(x_{0}\right) & =-1 / 12 \int d^{3} y d^{3} z\left\langle\bar{\psi}\left(x_{0}\right) \gamma_{5} \tau^{a} \psi\left(x_{0}\right) \bar{\zeta}(y) \gamma_{5} \tau^{a} \zeta(z)\right\rangle .
\end{aligned}
$$

These correlators are calculated with the spatial link matrices at $t=0$ and $L$ set to unity.

We run directly at $\kappa_{c}$, determined through the PCAC mass $m_{P C A C}(L / 2)$, where

$$
m_{P C A C}\left(x_{0}\right)=\frac{\frac{1}{2}\left(\partial_{0}+\partial_{0}^{*}\right) f_{A}\left(x_{0}\right)}{2 f_{P}\left(x_{0}\right)}
$$

and

$$
f_{A}\left(x_{0}\right)=-1 / 12 \int d^{3} y d^{3} z\left\langle\bar{\psi}\left(x_{0}\right) \gamma_{0} \gamma_{5} \tau^{a} \psi\left(x_{0}\right) \bar{\zeta}(y) \gamma_{5} \tau^{a} \zeta(z)\right\rangle .
$$

Here $\partial_{0}$ and $\partial_{0}^{*}$ are defined by $\partial_{0} f(x)=f(x+1)-f(x)$ and $\partial_{0}^{*} f(x)=f(x)-f(x-1)$. The correlators are calculated on lattices of size $L$ with the spatial link matrices at $t=0$ and $L$ set to unity.

We define $\kappa_{c}$ by the point where $m_{P C A C}$ vanishes on the $6^{4}$ and $8^{4}$ lattices, a linear extrapolation in $a / L$ from these values, and the values for $16^{4}$ lattices quoted in Ref. [8]. In practice we achieve $\left|a m_{\mathrm{PCAC}}\right| \lesssim 0.005$. We check explicitly that there is no residual sensitivity to the small remaining quark mass by repeating some of our simulations at $m_{\mathrm{PCAC}} \sim 0.02$.

\section{Evidence for fixed points}

Recent lattice studies have focused on the running of the SF coupling, emphasizing the slow running of this quantity $[9,10,11,8]$. These results have to be interpreted with care. Lattice data can find a range of energies over which no running is observed, but one cannot conclude that this extends to arbitrarily large distances, as one would expect in the presence of an IRFP. On the other hand, if the plateau has a finite extent, i.e. if the theory seems to walk, the behaviour of the running coupling depends on the scheme, and therefore the conclusions become less compelling.

There are instances where the beta function of an asymptotically free theory is numerically small. This is the case of the theory considered in this work in the perturbative regime. In this case, even though the theory does not have a fixed point, the running of the coupling is very slow. High accuracy is needed in order to resolve a "slow" running; therefore numerical studies of potential 
IRFP need high statistics, and a robust control of systematics. In particular it is important to extrapolate to the continuum limit to eliminate lattice artefacts.

Studies of the SF running coupling are a useful tool to expose the possible existence of theories that show a conformal behaviour at large distances. However the results have to be interpreted with care; they are unlikely to provide conclusive evidence about the existence of a fixed point by themselves. A more convincing picture can emerge when they are combined with spectral studies.

\section{Results for the coupling}

We have measured the coupling $\bar{g}^{2}(\beta, L)$ for a range of $\beta, L$. Our results are plotted in Fig. 1. They are directly comparable to those of Ref [8], and agree within statistical errors. It is clear that the coupling is very similar for different $L / a$ at a given value of $\beta$, and hence that it runs slowly.

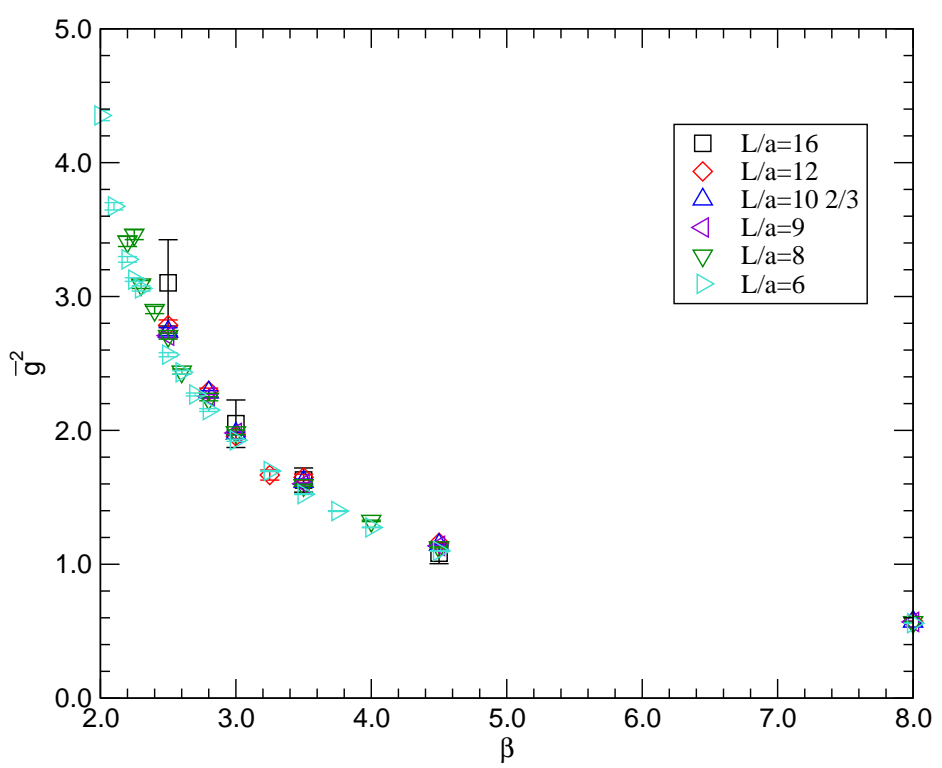

Figure 1: Data for the running coupling as computed from lattice simulations of the Schrödinger functional.

In order to see how the coupling runs we define the step scaling function $\sigma(u, s)$ as

$$
\sigma(u, s)=\left.\bar{g}^{2}(s L)\right|_{\bar{g}^{2}(L)=u}
$$

This is the continuum extrapolation of $\Sigma(u, s, a / L)$ which we calculate at various $a / L$.

We interpolate quadratically in $a / L$ to find values of $\bar{g}^{2}(\beta, L)$ at $L=9,10 \frac{2}{3}$, which gives us data for four steps of size $s=4 / 3$ for $L \rightarrow s L: L=6,8,9,12 ; s L=8,10 \frac{2}{3}, 12,16$. Then for each $\mathrm{L}$ we perform an interpolation in $\beta$. We can then find estimates of $\Sigma(u, 4 / 3, a / L)$ at any $u$. A continuum extrapolation is then performed in $a / L$ to give an estimate of $\sigma(u) \equiv \sigma(u, 4 / 3)$. The $L=6$ data was found to have large $O(a)$ artifacts, and we have too few $L=16$ points to constrain the interpolation functions, so neither are used in the continuum extrapolation.

The resulting values for $\sigma(u)$ can be seen in Fig. 2. The systematic errors from varying the interpolation functions or the continuum extrapolation were significantly larger than the statistical errors. To quantify this, we recalculated $\sigma(u)$ with a range of different interpolation and extrapolation functions. The resulting extremal values of $\sigma(u)$ were used as upper and lower bounds on the 
central value. The black error bars in Fig. 2 are determined in this way, but using only a constant continuum extrapolation. These values are consistent with a fixed point in the region $\bar{g}^{2} \sim 2.0-3.2$, as reported in Ref. [8]. The errors from also including the linear continuum extrapolation are much larger and mask any evidence for a fixed point, as also shown in Fig. 2.

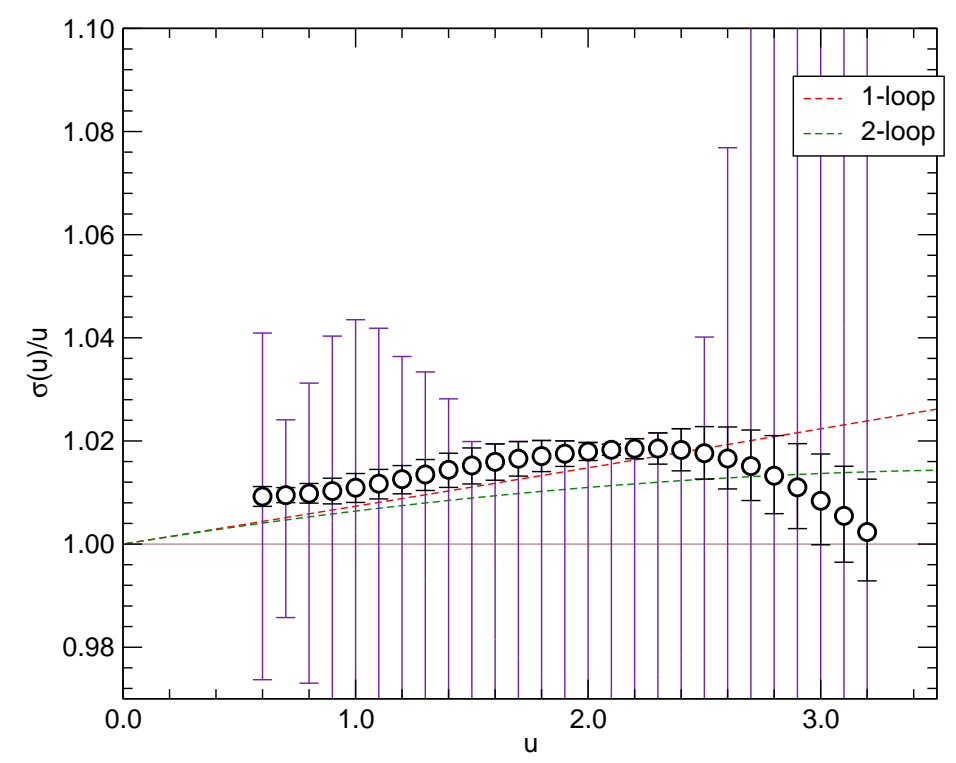

Figure 2: The relative step-scaling function $\sigma(u) / u$. The black circles use only a constant continuum extrapolation. The purple error bars include both constant and linear continuum extrapolations.

\section{Running mass}

We have measured $Z_{P}(\beta, L)$ for a range of $\beta, L$. We plot our results in Fig. 3, where we see a clear trend in $Z_{P}$ as a function of $L$ at all $\beta$. The step scaling function $\sigma_{P}(u, s)$ is then defined as:

$$
\sigma_{P}(u, s)=\left.\frac{Z_{P}(s L)}{Z_{P}(L)}\right|_{\bar{g}^{2}(L)=u}
$$

We extract this from a continuum extrapolation of $\Sigma_{P}(u, s, a / L)$.

The method for calculating $\sigma_{P}(u) \equiv \sigma_{P}(u, 4 / 3)$ is similar to that for calculating $\sigma(u)$. $Z_{P}$ converges faster than $\bar{g}^{2}$ and we have better $16^{4}$ data so here we use 4 points in our continuum extrapolations. Again the errors are dominated by systematics, in particular the choice of continuum extrapolation. We find good agreement with the 1-loop perturbative prediction.

We cannot determine directly the running of the mass with scale since we observe no running of the coupling within errors. However, we can define an estimator for the anomalous dimension,

$$
\gamma(u)=-\frac{\ln \left|\sigma_{P}(u, s)\right|}{\ln |s|}
$$

which is equal to the anomalous dimension at an IRFP, and which we plot in Fig. 4. We see that it is rather small over the range of interest; in particular, at $\bar{g}^{2}=2.2$, the benchmark value for the IRFP in [8], we have $\gamma=0.114_{-35}^{+78}$, and over the whole range $\bar{g}^{2}=2.0-3.2$ consistent with an IRFP in [8], we find $0.07<\gamma<0.56$. 


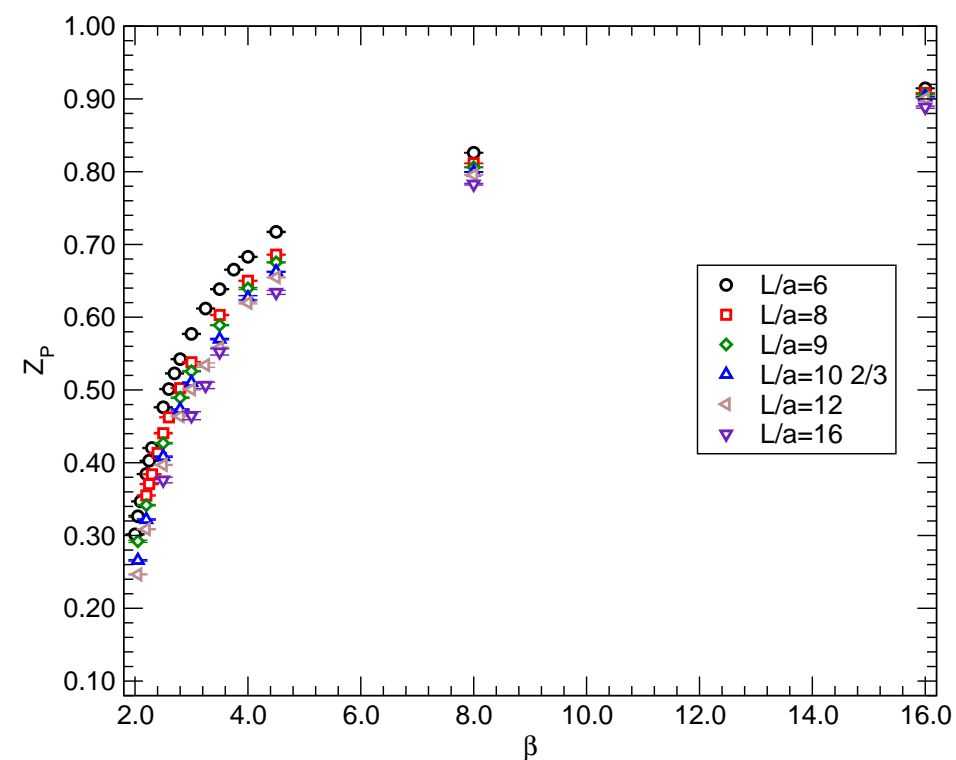

Figure 3: Data for the renormalisation constant $Z_{P}$ from lattice simulations of the Schrödinger functional.

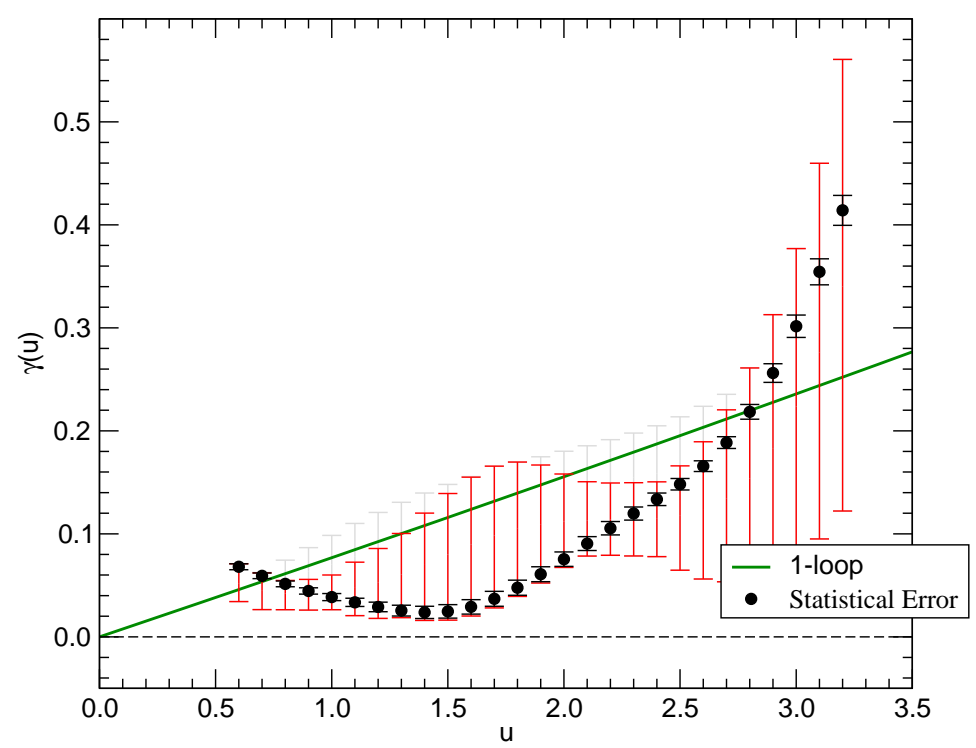

Figure 4: The mass anomalous dimension $\gamma(u)$. Red error bars use only a linear continuum extrapolation. Grey error bars include both constant and linear continuum extrapolations.

\section{Conclusions}

In these proceedings we have presented results for the running of the Schrödinger Functional coupling $\bar{g}^{2}$ and the mass anomalous dimension $\gamma$.

Our results for the running of the coupling are completely consistent with those of Ref. [8]. Our statistical errors are larger; however, we have carried out our analysis in a way that allows us to take the continnum limit with full control over the resulting systematic errors. Our results appear to show a slowing in the running of the coupling above $\bar{g}^{2}=2$ or so, and are consistent with the 
presence of a fixed point at somewhat higher $\bar{g}^{2}$. However, once we include the systematic errors from the continuum extrapolation that our results no longer give any evidence for a fixed point.

By contrast, we find that the behaviour of the anomalous dimension $\gamma$ is much easier to establish. We find a moderate anomalous dimension, close to the 1-loop perturbative prediction, throughout the range of $\beta$ explored. In particular, at $\bar{g}^{2}=2.2$, the benchmark value for the IRFP in [8], we find $\gamma=0.114_{-35}^{+78}$. This value is much smaller than that required for phenomenology, which is typically of order 1-2. Such large values of $\gamma$ are clearly inconsistent with our results.

The anomalous dimension is more vital than the running of $\bar{g}^{2}$ for phenomenology; if it is not large then the presence or absence of walking behaviour becomes academic. Hence the implications of our measurement of $\gamma$ for minimal walking technicolor deserve to be studied carefully.

The results presented here are preliminary, and the systematic errors need to be reduced to make our conclusions more robust. Using larger lattices would make the continuum extrapolations more accurate, and it may also be necessary to use an improved action to reach the precision required to show the existence of an IRFP or of walking behaviour. However this is very unlikely to affect our phenomenologically most important result, namely that $\gamma$ is not large.

\section{References}

[1] D. Dietrich and F. Sannino, Conformal window of $S U(N)$ gauge theories with fermions in higher dimensional representations, Phys. Rev. D75:085018, 2007.

[2] M. Luscher, P. Weisz, and U. Wolff, A Numerical method to compute the running coupling in asymptotically free theories, Nucl. Phys. B359:221, 1991.

[3] M. Luscher, R. Narayanan, P. Weisz, and U. Wolff, The Schrodinger functional: A Renormalizable probe for nonAbelian gauge theories, Nucl. Phys. B384:168, 1992.

[4] M. Luscher, R. Sommer, U. Wolff, and P. Weisz, Computation of the running coupling in the SU(2) Yang-Mills theory, Nucl. Phys. B389:247, 1993.

[5] S. Sint and R. Sommer, The Running coupling from the QCD Schrodinger functional: A One loop analysis, Nucl. Phys. B465:71, 1996.

[6] L. Del Debbio, A. Patella, and C. Pica, Higher representations on the lattice: numerical simulations. SU(2) with adjoint fermions, arXiv:0805.2058.

[7] S. Capitani, M. Luscher, R. Sommer, and H. Wittig, Non-perturbative quark mass renormalization in quenched lattice QCD, Nucl. Phys. B544:669, 1999.

[8] A. Hietanen, K. Rummukainen, and K. Tuominen, Evolution of the coupling constant in SU(2) lattice gauge theory with two adjoint fermions, arXiv:0904.0864.

[9] T. Appelquist, G. Fleming, and E. Neil, Lattice Study of the Conformal Window in QCD-like Theories, Phys. Rev. Lett. 100:171607, 2008.

[10] Y. Shamir, B. Svetitsky, and T. DeGrand, Zero of the discrete beta function in SU(3) lattice gauge theory with color sextet fermions, Phys. Rev. D78:031502, 2008.

[11] T. Appelquist, G. Fleming, and E. Neil, Lattice Study of Conformal Behavior in SU(3) Yang-Mills Theories, Phys. Rev. D79:076010, 2009. 